\title{
To Drop or Not to Drop: Receiver Design Principles for Estimation over Wireless Links
}

\author{
Yasamin Mostofi $^{\dagger}$ and Richard M. Murray ${ }^{\dagger \dagger}$ \\ ${ }^{\dagger}$ University of New Mexico ${ }^{\dagger \dagger}$ California Institute of Technology
}

\begin{abstract}
In this paper we consider estimation of a multipleinput multiple-output dynamical system over a wireless fading communication channel using a Kalman filter. We are interested in finding the optimum receiver design in terms of handing noisy samples. We reformulate the estimation problem to include the impact of stochastic communication noise in the noisy packets. We will show how the eigenvalues of the state transition matrix $A$ affect the optimum receiver design. We prove that, in the absence of a cross-layer information path, packet drop should be designed to balance information loss and communication noise in order to optimize the performance. In the presence of a cross-layer path, we show that keeping all the packets will minimize the average estimation error covariance. We also derive the stability condition in the presence of noisy packets and prove that it is independent of the shape of the communication noise variance or availability of a cross-layer information path.
\end{abstract}

\section{INTRODUCTION}

In this paper, we are interested in mobile sensor networks that are running real-time applications and are therefore delay-sensitive. We consider a mobile sensor that is observing a dynamical system. It transmits its observation over a wireless link to a remote node that is in charge of estimation using a Kalman filter. This is a fundamental problem that can arise in networked sensing, estimation and control.

Communication plays a key role in the overall performance of such networks since both sensor measurements and control commands are transmitted over wireless links. Digital transmission over wireless links can experience bit error rate due to multipath fading, shadowing, receiver thermal noise and excessive distance between the transmitter and receiver [1]. This is in addition to the impact of quantization. Impact of quantization on estimation and control has been studied extensively. The impact of other channel unreliability such as fading, shadowing, and mobility, however, has not been studied extensively. For mobile applications, such channel unreliability will introduce a time-varying noise in the received samples. The receiver can then decide to either keep the received sample or drop it. The criteria for making this decision vary depending on the application. Data networks, for example, are not as sensitive to delays since the application is not real time. The receiver, therefore, can afford to drop erroneous packets and wait for retransmission. Voice applications such as cellular networks, on the other hand, are sensitive to delays. In every transmitted bit stream, there are key bits embedded for synchronization and other crucial tasks. If these bits get corrupted, the receiver drops the transmitted stream. However, once these bits are received accurately, the rest of the bit error rate is either corrected through channel coding or tolerated [2] since there is no time for retransmission. Estimation and control of dynamical systems over wireless links is an emerging application, for which new communication design paradigms should be developed. Control applications are typically delay sensitive as we are racing against the dynamics of the system. Therefore, the communication protocols and designs suitable for other already-existing applications like data networks may not be entirely applicable.

Current work in literature has assumed applying data network design principles to networked control applications by considering a receiver that only keeps noise-free packets (packet erasure channels). Along this line, impact of packet drop on networked control applications has been studied. Micheli et al. investigated impact of packet loss on estimation by considering random sampling of a dynamical system [3]. This is followed by the work of Sinopoli et al. which derived bounds for the maximum tolerable probability of packet loss to maintain stability [4]. In [5], [6] we studied the impact of the stochastic communication noise introduced by fading and mobility on networked control systems. Instead of applying data network design principles to such delay-sensitive applications, we developed new receiver design paradigms for single-input single-output systems. In this paper, we are extending that work to multiple-input multiple-output cases. The main question this paper addresses is the following: "How should noisy packets be handled in the receiver for a multiple-input multiple-output system?". We answer this question both in terms of stability and performance. We will find the impact of the eigenvalues of matrix $A$. Furthermore, we explore the role of a cross-layer information path and its impact on the optimum design.

\section{System MODEL}

Consider a mobile sensor observing a linear dynamical system: $x[k+1]=A x[k]+w[k]$ and $y[k]=C x[k]+v[k]$, where $x[k] \in \mathbb{R}^{N}$ and $y[k] \in \mathbb{R}^{M}$ represent the state and observation respectively. $w[k] \in \mathbb{R}^{N}$ and $v[k] \in \mathbb{R}^{M}$ represent zero-mean Gaussian process and observation noise vectors with covariances of $Q \succcurlyeq 0$ and $R \succ 0$ respectively. In this paper, we take $M=N$ and $C$ invertible to focus on the impact of communication noise. We are interested in estimating unstable dynamics and therefore we consider cases where matrix $A$ has at least one eigenvalue outside the unit circle. The sensor then transmits its observation over a wireless fading channel to a remote node, which is in charge of estimation. Since estimation of dynamical systems over 
mobile links has not been extensively studied before, we keep our analysis general by considering mobile channels.

\section{A. Physical Layer: Wireless Communication [1]}

In this part we will summarize how to model the impact of a time-varying fading wireless communication channel on the observation. The sensor quantizes the observation, $y[k]$, transforms it into a packet of bits and transmits it over a fading channel. The remote node will receive a noisy version of the transmitted data. Let $\hat{y}[k]$ represent the signal at the end of the physical layer pipeline. $\hat{y}[k]$ is what the second node assumes the $k^{t h}$ transmitted observation was. Let $n[k]$ represent the difference between the transmitted observation and the received one at the end of the physical layer pipeline: $n[k]=y[k]-\hat{y}[k]$, where $n[k]=n_{c}[k]+n_{q}[k]$. In this paper, we refer to $n[k]$ as communication noise. It consists of two parts, link noise $\left(n_{c}\right)$ : noise due to the quality of the communication link and quantization noise $\left(n_{q}\right)$. Since the impact of quantization noise on estimation and control is studied extensively, in this paper we are mainly interested in the impact of the link noise.

1) Multipath Fading Channel: One of the major performance degradation factors of a mobile communication environment is multipath fading. "Multipath" is a term used to describe multiple paths that a radio wave may follow between the transmitter and the receiver. Waves that are received in phase reinforce each other producing a stronger signal, while those that are received out of phase produce a weaker signal. Small changes in the transmission paths, caused by movements of the receiver or transmitter can change the phase relationship of the two signals, introducing a rapidly time-varying fading channel. This is in addition to the distance-dependent attenuation factor.

2) Channel Signal to Noise Ratio: A fundamental parameter that characterizes the performance of a communication channel is the received Signal to Noise Ratio. Received Signal to Noise Ratio is defined as the ratio of the received signal power divided by the receiver thermal noise power. Let $\Upsilon[k]$ represent the instantaneous received Signal to Noise Ratio at $k^{\text {th }}$ transmission. $\Upsilon[k]$ determines how well the transmitted bits of the $k^{t h}$ transmission can be retrieved. As the sensor moves, the remote node will experience different channels and therefore different received Signal to Noise Ratios. In a given area, $\Upsilon[k]$ can be considered a stationary stochastic process with $\Upsilon_{\text {ave }}$ representing its average. The distribution of $\Upsilon[k]$ is a function of the transmission environment and the level of mobility of the sensor. In this paper we do not make any assumption on the probability distribution of $\Upsilon$. Only when we want to provide an example, we will take $\Upsilon$ to be exponentially distributed, which is a common model for outdoor fading channels with no Line-Of-Sight path. We also take $\Upsilon$ to be uncorrelated from one transmission to the next. This will be the case as long as the time interval between consecutive transmissions is bigger than channel coherence time [1].

3) Communication Noise Variance: Let $\sigma_{n}^{2}[k]$ represent the variance of $n[k]$ at $k^{t h}$ transmission: $\sigma_{n}^{2}[k]=$
$\mathbb{E}\left(n^{2}[k] \mid \Upsilon[k]\right)=\Xi(\Upsilon[k])$. When necessary, we may write $\sigma_{n}^{2}(\Upsilon)$ to indicate the dependency. $\Xi$ is a non-increasing function that depends on the transmitter and receiver design principles, such as modulation and coding, as well as the transmission environment. To keep our analysis general, in this paper we do not make any assumption on $\Xi$.

4) Packet Drop Probability: Depending on the receiver design, there can be a packet drop mechanism deployed in the receiver. Let $\mu[k]$ represent the probability that the receiver drops the $k^{t h}$ packet. $\mu[k]$ can also be represented as a function of $\Upsilon[k]: \mu[k]=G(\Upsilon[k])$, where function $G$ is a non-increasing function. Functions $\Xi$ and $G$ provide the abstraction necessary to consider the impact of physical layer in the higher application layer. To ease mathematical derivations, in this paper we will approximate function $G$ with the following:

$$
\mu[k]=\left\{\begin{array}{cc}
0 & \Upsilon[k] \geq \Upsilon_{T} \\
1 & \text { else }
\end{array}\right.
$$

This means that the receiver keeps those packets with received Signal to Noise Ratio above a designated threshold: $\Upsilon_{T}$. Experimental results confirm this to be a good approximation [7].

\section{B. Application Layer: Estimation}

The remote node estimates the state based on the received observation using a Kalman filter [8]. Let $\hat{x}[k]$ denote the estimate of $x[k]$ at the remote node. Then $P[k]$ represents the corresponding estimation error covariance matrix given $\Upsilon[k-1], \Upsilon[k-2], \ldots, \Upsilon[0]$ :

$P[k]=\mathbb{E}\left[(x[k]-\hat{x}[k])(x[k]-\hat{x}[k])^{T}\right]_{\mid \Upsilon[k-1], \Upsilon[k-2], \ldots, \Upsilon[0]}$.

This is different from traditional form of Kalman filtering since $P[k]$ is a function of channel statistics through $\Upsilon[k-1]$, $\Upsilon[k-2], \ldots, \Upsilon[0]$. To obtain $\mathbb{E}(P[k]), P[k]$ should be averaged over the joint distribution of $\Upsilon[k-1], \Upsilon[k-2]$, $\ldots, \Upsilon[0]$. There will be different forms of recursion for $P[k]$ depending on the availability of a cross-layer information path in the receiver, as we shall see in the subsequent sections.

\section{Cross-Layer Information Path}

When estimating over wireless links, the application layer will be in charge of estimation whereas the knowledge of the quality of the communication link will be available in the physical layer. A cross-layer information path in this paper refers to a path from physical layer to the application layer that carries information on the quality of the link (Signal to Noise Ratio or communication noise variance). In other words, the physical layer can let the application layer know, using a cross-layer path, how much it trusts the accuracy of each received packet. While presence of such a path can play a key role in the overall performance and the optimum design typically involves one, the receiver architecture may not support it. Therefore, we will consider scenarios where such a path is available at the receiver as well as cases where it is not supported by the architecture. 


\section{Scenario 1: Ideal Communication Noise}

As discussed in Section I, current work in literature mainly applies data network design principles to networked control applications by assuming that the receiver drops packets that contain any amount of error. Then those packets that are kept in the receiver are considered noise-free. We refer to this assumption on the communication noise as "ideal noise" throughout the paper. Similarly we refer to this design strategy, which applies data-network type protocols, as "scenario 1 ". Such an assumption translates to the following recursion for the estimation error covariance:

$$
\begin{gathered}
P[k+1]=A P[k] A^{T}+Q \\
-A P[k] C^{T}\left(R+C P[k] C^{T}+S_{1}(\Upsilon[k])\right)^{-1} C P[k] A^{T},
\end{gathered}
$$

where $S_{1}[k]=\left\{\begin{array}{cc}0 & \Upsilon[k] \geq \Upsilon_{T} \\ \infty & \text { otherwise }\end{array}\right.$. Current work in literature mainly considers Kalman filtering over fixed wireless channels by assuming that the probability of packet drop is the same from one transmission to the next. For a fixed probability of packet drop, $\mu_{\text {scenario } 1}$, authors in [4] found the following condition for stability: $\mu_{\text {scenario } 1}<\rho_{\max }^{-2}$, where $\rho_{\max }$ represents spectral radius of matrix $A$.

\section{RECEIVER DESIGN THEORIES}

In Section II-D we saw that the current work in the literature assumed a receiver that drops those packets that contain any amount of error, which is a suitable design for non real-time applications like data networks. Estimation of a rapidly changing dynamical system, however, is delay sensitive. Dropping all the erroneous packets can result in loss of information, can reduce the useful transmission rate and can lead to instability. In this section, we will consider the impact of stochastic communication noise on the estimation of a multiple-input multiple-output system and will derive receiver design theories for real-time estimation. To keep the analysis general, we will not make any assumption on the communication noise variance or Signal to Noise Ratio distribution. We will analyze the performance and stability conditions for the following cases:

Scenario 2: Receiver can not provide a cross-layer path,

Scenario 3: Receiver is equipped with a cross-layer path.

\section{A. Scenario 2: Case of No Cross-Layer Path}

In this part we will consider a receiver that does not support a cross-layer path. Then the application layer (i.e. the Kalman filter) does not have any knowledge of the quality of the communication link. We will find the optimum way of dropping packets for such a receiver. To ease mathematical derivation of this scenario, we assume that the observation noise is negligible compared to the communication noise. ${ }^{1}$

\footnotetext{
${ }^{1}$ The analysis can be similarly carried out under the condition that the knowledge of observation noise covariance, $R$, is not available in the estimator. Then $\sigma_{n}^{2} I_{N}$ should be replaced by $\sigma_{n}^{2} I_{N}+R$ throughout the analysis.
}

The estimation using a Kalman filter will then be as follows:

$$
\hat{x}[k+1]=\left\{\begin{array}{cc}
A \hat{x}[k] & \text { if } k^{t h} \text { packet is dropped } \\
A C^{-1} \hat{y}[k] & \text { if } k^{t h} \text { packet is kept. }
\end{array}\right.
$$

The estimation error will be as follows:

$$
\begin{gathered}
x[k+1]-\hat{x}[k+1]= \\
\left\{\begin{array}{cc}
A(x[k]-\hat{x}[k])+w[k] & \text { if } k^{t h} \text { packet is dropped } \\
w[k]-A C^{-1}(v[k]+n[k]) & \text { if } k^{t h} \text { packet is kept. }
\end{array}\right.
\end{gathered}
$$

This will result in the following recursion for the estimation error covariance, assuming that the observation noise is negligible:

$$
\begin{gathered}
P[k+1]= \\
A P[k] A^{T}+Q-\frac{A P[k] A^{T}-A C^{-1} \sigma_{n}^{2}[k] C^{-1 T} A^{T}}{S_{2}[k]},
\end{gathered}
$$

where $\sigma_{n}^{2}$ is the communication noise variance as defined in Section II and $S_{2}[k]=\left\{\begin{array}{cc}1 & \Upsilon[k] \geq \Upsilon_{T} \\ \infty & \text { otherwise. }\end{array}\right.$. As the mobile node moves in a given area, it will experience different Signal to noise Ratios. Averaging Eq. 6 over $\Upsilon[k], \Upsilon[k-1], \ldots$ will result in the following recursion for average estimation error covariance:

$$
\begin{gathered}
\mathbb{E}(P[k+1])= \\
\mu_{\text {ave }}\left(\Upsilon_{T}\right) A \mathbb{E}(P[k]) A^{T}+Q+\sigma_{n, \text { ave }}^{2}\left(\Upsilon_{T}\right) A\left(C^{T} C\right)^{-1} A^{T} .
\end{gathered}
$$

$\mu_{\text {ave }}$ and $\sigma_{n, a v e}^{2}$ represent average probability of packet loss (spatial averaging) and average communication noise variance that entered the estimation process respectively:

$$
\mu_{\text {ave }}\left(\Upsilon_{T}\right)=\mathbb{E}(\mu)=\int_{0}^{\Upsilon_{T}} \chi(\Upsilon) d \Upsilon
$$

and

$$
\sigma_{n, a v e}^{2}\left(\Upsilon_{T}\right)=\int_{\Upsilon_{T}}^{\infty} \sigma_{n}^{2}(\Upsilon) \chi(\Upsilon) d \Upsilon
$$

where $\chi$ represents probability density function of $\Upsilon$.

Lemma 1 (see [8]): Consider the following Lyapunov equation with $\Theta$ Hermitian and $\Pi$ real: $\Sigma=\Pi \Sigma \Pi^{T}+\Theta$. The following hold:

a) If $\Pi$ is a stable matrix (spectral radius less that one), $\Sigma$ will be unique and Hermitian and can be expressed as follows: $\Sigma=\sum_{i=0}^{\infty} \Pi^{i} \Theta\left(\Pi^{T}\right)^{i}$,

b) if $\left\{\Pi, \Theta^{1 / 2}\right\}$ is controllable and $\Theta \succcurlyeq 0$, then $\Sigma$ will be Hermitian, unique and positive-definite iff $\Pi$ is stable.

\section{1) Stability:}

Definition 1: We consider the estimation process stable as long as average estimation error covariance stays bounded.

Using Lemma $1 b$, it can be easily seen from Eq. 7 that the stability condition will be as follows:

$$
\mu_{\text {ave,scenario } 2}<\rho_{\max }^{-2},
$$

where $\rho_{\max }$ represents the spectral radius of matrix $A$.

Remark 1: The stability condition is independent of the shape of the communication noise variance, $\sigma_{n}^{2}$.

2) Optimum Performance: Intuitively, there should be an optimum $\Upsilon_{T}$ (optimum way of dropping packets) that will minimize the asymptotic average estimation error covariance 
for this case. If $\Upsilon_{T}$ is too low, the receiver will keep most of the packets but the estimation will be too noisy. On the other hand, if $\Upsilon_{T}$ is too high, the receiver will be strict about the quality of the packets that it will keep. This reduces the amount of communication noise that enters the estimation process but will result in high packet loss rate and therefore information loss rate. Then the optimum $\Upsilon_{T}$ will be the one that provides a balance between information loss and communication noise. In [6], we related the optimum $\Upsilon_{T}$ to the dynamics of the system under estimation for a scalar case. Here we will find how eigenvalues of $A$ affect the optimum design.

The asymptotic average estimation error covariance is as follows as long as the stability condition of Eq. 10 holds:

$$
\begin{gathered}
\mathbb{E}(P[\infty])=\mu_{\text {ave }}\left(\Upsilon_{T}\right) A \mathbb{E}(P[\infty]) A^{T}+ \\
\sigma_{n, a v e}^{2}\left(\Upsilon_{T}\right) A\left(C^{T} C\right)^{-1} A^{T}+Q \text { for } \mu_{\text {ave }}\left(\Upsilon_{T}\right)<\rho_{\max }^{-2} .
\end{gathered}
$$

Let $\Upsilon_{T_{1} \text {,opt }}$ represent the optimum way of dropping packets which will minimize the spectral norm of the asymptotic average estimation error covariance matrix:

$$
\Upsilon_{T_{1}, \text { opt }}=\arg \min \left\|\mathbb{E}\left(P\left[\infty, \Upsilon_{T}\right]\right)\right\|
$$

Let $\Upsilon_{T_{2} \text {,opt }}$ represent the optimum way of dropping packets which will minimize the determinant of the asymptotic average estimation error covariance:

$$
\Upsilon_{T_{2}, o p t}=\arg \min \operatorname{det} \mathbb{E}\left(P\left[\infty, \Upsilon_{T}\right]\right)
$$

We will derive an analytical expression that relates optimum way of dropping packets to the characteristics of the communication channel and eigenvalues of matrix $A$. For this derivation, we assume that $C=\varsigma I_{N}$ and $Q=q I_{N}$, where $I_{N}$ represents an $N \times N$ identity matrix. We furthermore take $A=A_{s}$, where $A_{s}$ is a symmetric matrix, i.e. $A_{s}=A_{s}^{T}$. Later in this subsection we will discuss cases where $A$ is not symmetric.

Theorem 1 (Balance of Information Loss \& Communication Noise): Consider the system model described in Section II, with $C=\varsigma I_{N}, Q=q I_{N}$ and $A=A_{s}$. Consider a receiver that is equipped with a packet drop mechanism described by Eq. 1 and does not support a cross-layer path. Then $\Upsilon_{T_{1} \text {,opt }}$ will be as follows:

$$
\Upsilon_{T_{1}, o p t}=\left\{\begin{array}{cc}
\Upsilon_{T_{1}}^{*} & \Upsilon_{T_{1}}^{*} \geq 0 \\
0 & \text { otherwise }
\end{array}\right.
$$

where $\Upsilon_{T_{1}}^{*}$ is the unique solution to the following equation:

$$
\underbrace{\mu_{\text {ave }}\left(\Upsilon_{T_{1}}^{*}\right)}_{\text {information loss }}+\underbrace{\sigma_{n, \text { norm }}^{2}\left(\Upsilon_{T_{1}}^{*}\right)}_{\text {communication noise }}+\frac{\varsigma^{2} q}{\rho_{\max }^{2} \sigma_{n}^{2}\left(\Upsilon=\Upsilon_{T_{1}}^{*}\right)}=\rho_{\max }^{-2}
$$

where $\sigma_{n, \text { norm }}^{2}$ refers to the normalized average communication noise variance: $\sigma_{n, \text { norm }}^{2}\left(\Upsilon_{T_{1}}^{*}\right)=\frac{\sigma_{n, \text { ave }}^{2}\left(\Upsilon_{T_{1}}^{*}\right)}{\sigma_{n}^{2}\left(\Upsilon=\Upsilon_{T_{1}}^{*}\right)}$, and $\Upsilon_{T_{2} \text {,opt }}$ will be as follows:

$$
\Upsilon_{T_{2}, o p t}=\left\{\begin{array}{cc}
\Upsilon_{T_{2}}^{*} & \Upsilon_{T_{2}}^{*} \geq 0 \\
0 & \text { otherwise }
\end{array}\right.
$$

where $\Upsilon_{T_{2}}^{*}$ is the unique solution to the following equation:

$$
\sum_{i=1}^{N} \frac{\rho_{i}^{2}}{1-\rho_{i}^{2} \mu_{\text {ave }}\left(\Upsilon_{T_{2}}^{*}\right)}=\sum_{i=1}^{N} \frac{1}{\sigma_{n, \text { norm }}^{2}\left(\Upsilon_{T_{2}}^{*}\right)+\frac{q \varsigma^{2}}{\sigma_{n}^{2}\left(\Upsilon=\Upsilon_{T_{2}}^{*}\right) \rho_{i}^{2}}}
$$

where $\rho_{1}, \rho_{2}, \ldots, \rho_{N}$ represent eigenvalues of matrix $A$, where $\left|\rho_{1}\right| \geq\left|\rho_{2}\right| \geq \ldots \geq\left|\rho_{N}\right|$ and $\rho_{\max }=\left|\rho_{1}\right|$.

Proof of Theorem 1: Using Lemma la (under stability condition of Eq. 10) with $C=\varsigma I_{N}, Q=q I_{N}$ and $A=A_{s}$ will result in

$$
\begin{aligned}
\mathbb{E}(P[\infty])= & \varsigma^{-2} \sigma_{n, a v e}^{2}\left(\Upsilon_{T}\right) \sum_{i=0}^{\infty} \mu_{\text {ave }}^{i}\left(\Upsilon_{T}\right)\left(A_{s}\right)^{2 i+2} \\
& +q \sum_{i=0}^{\infty} \mu_{\text {ave }}^{i}\left(\Upsilon_{T}\right)\left(A_{s}\right)^{2 i}
\end{aligned}
$$

We will have the following decomposition: $A_{s}=L \Lambda L^{T}$, where $\Lambda=\operatorname{diag}\left\{\rho_{1}, \rho_{2}, \ldots, \rho_{N}\right\}$ and $L^{T} L=I_{N}$. It can be confirmed that,

$$
\begin{gathered}
\mathbb{E}(P[\infty])= \\
\operatorname{Ldiag}\left\{\frac{q+\varsigma^{-2} \rho_{1}^{2} \sigma_{n, a v e}^{2}\left(\Upsilon_{T}\right)}{1-\rho_{1}^{2} \mu_{\text {ave }}\left(\Upsilon_{T}\right)}, \ldots, \frac{q+\varsigma^{-2} \rho_{N}^{2} \sigma_{n, a v e}^{2}\left(\Upsilon_{T}\right)}{1-\rho_{N}^{2} \mu_{\text {ave }}\left(\Upsilon_{T}\right)}\right\} L^{T},
\end{gathered}
$$

This results in

$$
\|\mathbb{E}(P[\infty])\|=\left(\frac{q+\varsigma^{-2} \rho_{1}^{2} \sigma_{n, a v e}^{2}\left(\Upsilon_{T}\right)}{1-\rho_{1}^{2} \mu_{a v e}\left(\Upsilon_{T}\right)}\right) .
$$

Let $\Upsilon_{T_{1}}^{*}$ represent any solution to Eq. 15 . It can be easily verified that $\frac{\partial E(\|P[\infty]\|)}{\partial \Upsilon_{T}}$ is only zero at $\Upsilon_{T_{1}}^{*}$. Next we show that Eq. 15 has a unique solution. Assume that Eq. 15 has two solutions: $\Upsilon_{T_{1,1}}^{*}$ and $\Upsilon_{T_{1,2}}^{*}>\Upsilon_{T_{1,1}}^{*}$. Since $\sigma_{n}^{2}$ is a nonincreasing function of $\Upsilon$, we will have the following:

$$
\begin{aligned}
& \mu_{\text {ave }}\left(\Upsilon_{T_{1,1}}^{*}\right)+\sigma_{n, \text { norm }}^{2}\left(\Upsilon_{T_{1,1}}^{*}\right)+\frac{\varsigma^{2} q}{\rho_{\max }^{2} \sigma_{n}^{2}\left(\Upsilon=\Upsilon_{T_{1,1}}^{*}\right)}- \\
& {\left[\mu_{\text {ave }}\left(\Upsilon_{T_{1,2}}^{*}\right)+\sigma_{n, \text { norm }}^{2}\left(\Upsilon_{T_{1,2}}^{*}\right)+\frac{\varsigma^{2} q}{\rho_{\max }^{2} \sigma_{n}^{2}\left(\Upsilon=\Upsilon_{T_{1,2}}^{*}\right)}\right]=} \\
& \underbrace{\int_{\Upsilon_{T_{1,2}}^{*}}^{\Upsilon_{T_{1,1}}^{*}} \chi(\Upsilon) d \Upsilon+\int_{\Upsilon_{T_{1,1}}^{*}}^{\Upsilon_{T_{1,2}}^{*}} \frac{\sigma_{n}^{2}(\Upsilon) \chi(\Upsilon)}{\sigma_{n}^{2}\left(\Upsilon=\Upsilon_{T_{1,1}}^{*}\right)} d \Upsilon}_{<0}+ \\
& \underbrace{\left(\frac{1}{\sigma_{n}^{2}\left(\Upsilon=\Upsilon_{T_{1,1}}^{*}\right)}-\frac{1}{\sigma_{n}^{2}\left(\Upsilon=\Upsilon_{T_{1,2}}^{*}\right)}\right) \int_{\Upsilon_{T_{1,2}}^{*}}^{\infty} \sigma_{n}^{2}(\Upsilon) \chi(\Upsilon) d \Upsilon}_{<0} \\
& +\underbrace{\frac{\varsigma^{2} q}{\rho_{\max }^{2}}\left(\frac{1}{\sigma_{n}^{2}\left(\Upsilon=\Upsilon_{T_{1,1}}^{*}\right)}-\frac{1}{\sigma_{n}^{2}\left(\Upsilon=\Upsilon_{T_{1,2}}^{*}\right)}\right)}_{<0}<0 .
\end{aligned}
$$

Therefore ${ }^{2}, \Upsilon_{T_{1,1}}^{*}=\Upsilon_{T_{1,2}}^{*}$. Let $\Upsilon_{T}^{c}$ be the critical stability threshold: $1-\rho_{\max }^{2} \mu_{\text {ave }}\left(\Upsilon_{T}^{c}\right)=0$. We have $\Upsilon_{T_{1}}^{*}<\Upsilon_{T}^{c}$. Consider those cases where there exists a positive solution to Eq. 15. Then using the fact that $\lim _{\Upsilon_{T} \rightarrow \Upsilon_{T}^{c}} \mathbb{E}\left(P\left[\infty, \Upsilon_{T}\right]\right) \rightarrow \infty$ shows that $\Upsilon_{T_{1}}^{*}$ corresponds to the unique minimum of $\left\|\mathbb{E}\left(P\left[\infty, \Upsilon_{T}\right]\right)\right\|$, i.e. $\Upsilon_{T_{1} \text { opt }}=\Upsilon_{T_{1}}^{*}$. If process noise is the dominant noise, compared to the communication noise, there may be no positive solution to Eq. 15. It can be easily seen that, in

${ }^{2}$ Note that $\frac{\partial \sigma_{n}^{2}(\Upsilon)}{\partial \Upsilon}$ is taken to be zero only asymptotically. 
such cases, $\left\|\mathbb{E}\left(P\left[\infty, \Upsilon_{T}\right]\right)\right\|$ will be an increasing function for $\Upsilon_{T} \geq 0$, resulting in $\Upsilon_{T_{1}, \text { opt }}=0$.

Next we will find $\Upsilon_{T_{2}, \text { opt }}$. We will have,

$$
\operatorname{det} \mathbb{E}(P[\infty])=\prod_{i=1}^{N} \frac{\rho_{i}^{2} \varsigma^{-2} \sigma_{n, a v e}^{2}\left(\Upsilon_{T}\right)+q}{1-\rho_{i}^{2} \mu_{\text {ave }}\left(\Upsilon_{T}\right)} .
$$

It can be easily confirmed that

$$
\begin{gathered}
\frac{\partial \operatorname{det} \mathbb{E}(P[\infty])}{\partial \Upsilon_{T}}=\chi\left(\Upsilon_{T}\right) \frac{\prod_{i=1}^{N}\left(\varsigma^{-2} \sigma_{n, a v e}^{2}\left(\Upsilon_{T}\right) \rho_{i}^{2}+q\right)}{\prod_{i=1}^{N}\left(1-\rho_{i}^{2} \mu_{a v e}\left(\Upsilon_{T}\right)\right)} \\
\times\left[\sum_{j=1}^{N} \frac{\rho_{j}^{2}}{1-\rho_{j}^{2} \mu_{a v e}\left(\Upsilon_{T}\right)}-\sum_{j=1}^{N} \frac{\varsigma^{-2} \sigma_{n}^{2}\left(\Upsilon=\Upsilon_{T}\right) \rho_{j}^{2}}{\varsigma^{-2} \sigma_{n, a v e}^{2}\left(\Upsilon_{T}\right) \rho_{j}^{2}+q}\right] .
\end{gathered}
$$

Therefore, $\frac{\partial \operatorname{det}(\mathbb{E}(P[\infty]))}{\partial \Upsilon_{T}} \mid \Upsilon_{T}=\Upsilon_{T_{2}}^{*}=0$ will result in Eq. 17. In a similar manner, it can be easily confirmed that Eq. 17 has a unique solution and that $\Upsilon_{T_{2} \text { opt }}$ corresponds to the global minimum of the determinant of the asymptotic average estimation error covariance.

Theorem 1 shows that the optimum way of dropping packets is the one that provides a balance between information loss $\left(\mu_{\text {ave }}\right)$ and communication noise $\left(\sigma_{n, a v e}^{2}\right)$. Eq. 15 (and Eq. 17) may not have a positive solution if process noise is the dominant noise compared to the communication noise (the third term on the left hand side of Eq. 15, for instance, can get considerably high values). In such cases, the receiver should keep all the packets as communication noise is not the bottleneck. However, as long as process noise is not the dominant noise, the optimum way of dropping packets is the one that provides a balance between information loss and communication noise.

Theorem 1 confirms that dropping all the erroneous packets will not minimize the estimation error covariance and that the optimum receiver would allow some amount of communication noise in the estimation process in order to avoid high information loss rate. In general, minimizing spectral norm and determinant would result in different optimum packet drop thresholds depending on the eigenvalues of matrix $A$, as can be seen from Theorem 1. If $A$ has one dominant eigenvalue or all the eigenvalues of $A$ are the same, then it is easy to see from Eq. 17 that $\Upsilon_{T_{1}, \text { opt }}=\Upsilon_{T_{2} \text {,opt }}$.

To see the impact of operating at the optimum $\Upsilon_{T}$, Fig. 1 shows $\|\mathbb{E}(P[\infty])\|$ as a function of $\Upsilon_{T}$ and for different levels of average Signal to Noise Ratio, $\Upsilon_{\text {ave }}$. For this example, Signal to Noise Ratio, $\Upsilon$, is taken to have an exponential distribution and the communication noise variance is taken as follows: $\sigma_{n}^{2}(\Upsilon)=\alpha+\delta \times \Omega(\sqrt{\Upsilon})$, where $\Omega(d)=$ $\frac{1}{\sqrt{2 \pi}} \int_{d}^{\infty} e^{-t^{2} / 2} d t$ for an arbitrary $d$. This is the variance of the communication noise for a binary modulation system that utilizes gray coding [9]. The following parameters are chosen for this example: $A=\left(\begin{array}{ccc}2 & 0.3 & 0.45 \\ 0.4 & 0.2 & 0.5 \\ 1.5 & 0.6 & 0.34\end{array}\right), Q=q I_{3}$, $C=\varsigma I_{3}, q=0.001, \varsigma=2, \alpha=1.27 \times 10^{-4}$ and $\delta=533.3$ (which corresponds to 10 bits per sample and quantization step size of 0.0391). It can be seen from Fig. 1 that if $\Upsilon_{T}$ is too low, estimation performance degrades due to excessive communication noise. On the other hand, having $\Upsilon_{T}$ too high will result in loss of information, which will degrade the performance. The optimum $\Upsilon_{T}$ (as predicted by Theorem 1) provides the necessary balance between loss of information and communication noise, reaching the minimums of the estimation error curves. As $\Upsilon_{T}$ increases, the estimation will approach the instability regions, predicted by Eq. 10 due to high information loss.

Remark 2: Eq. 15 is derived for symmetric $A$ matrices. Still, the minimums of the curves in Fig. 1 (optimum $\Upsilon_{T}$ ) satisfy Eq. 33 . This suggests that a similar expression could be valid for the general case. We will further discuss this in Section IV.

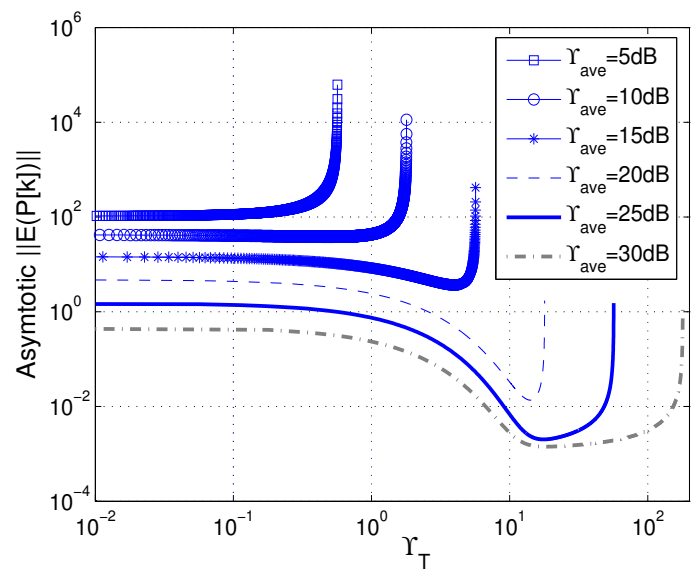

Fig. 1. Scenario 2: Minimums of the curves indicating optimum packet drop mechanism in the absence of a cross-layer path

\section{B. Scenario 3: Impact of A Cross-Layer Information Path}

Consider a scenario where the receiver can support a crosslayer path. This means that the Kalman filter will have access to and can utilize the knowledge of the communication noise variance. We will have the following recursion for the estimation error covariance:

$$
\begin{gathered}
P[k+1]=A P[k] A^{T}+Q \\
-A P[k] C^{T}\left(\sigma_{z}^{2}(\Upsilon[k])+C P[k] C^{T}\right)^{-1} C P[k] A^{T},
\end{gathered}
$$

where

$$
\sigma_{z}^{2}(\Upsilon[k])=\left\{\begin{array}{cc}
\sigma_{n}^{2}(\Upsilon[k]) I_{N}+R & \Upsilon[k] \geq \Upsilon_{T} \\
\infty & \text { otherwise }
\end{array}\right.
$$

\section{1) Stability}

Matrix Convexity (see [10]): Let $f$ represent a symmetric matrix-valued function, $f: \mathbb{R}^{N \times N} \rightarrow \mathbb{R}^{M \times M}$. Function $f$ is convex with respect to matrix inequality if

$$
f\left(\theta \Pi_{1}+(1-\theta) \Pi_{2}\right) \preccurlyeq \theta f\left(\Pi_{1}\right)+(1-\theta) f\left(\Pi_{2}\right),
$$

for arbitrary $\Pi_{1} \in$ and $\Pi_{2} \in \mathbb{R}^{N \times N}$ and $\theta \in[0,1]$.

Lemma 2: Consider $\Pi_{1}, \Pi_{2}, \Pi_{3}$ and $\Pi_{4} \in \mathbb{R}^{N \times N}$. The following can be easily confirmed (see [11]):

a) if $\Pi_{1}$ and $\Pi_{2}$ are positive definite, then

$\Pi_{1} \preccurlyeq \Pi_{2}$ if and only if $\Pi_{2}^{-1} \preccurlyeq \Pi_{1}^{-1}$.

b) if $0 \preccurlyeq \Pi_{1} \preccurlyeq \Pi_{2}$ and $0 \preccurlyeq \Pi_{3} \preccurlyeq \Pi_{4}$, then $\Pi_{1} \Pi_{3} \preccurlyeq$ $\Pi_{2} \Pi_{4}$. 
Lemma 3: Let $\Pi_{1}$ and $\Pi_{2} \in \mathbb{R}^{N \times N}$ represent symmetric positive definite matrices.

a) Let $f: \mathbb{R}^{N \times N} \rightarrow \mathbb{R}^{N \times N}$ represent inverse of $\Pi$ : $f(\Pi)=\Pi^{-1} . f$ is convex with respect to matrix inequality (see [12]).

b) if $f: \mathbb{R}^{N \times N} \rightarrow \mathbb{R}^{M \times M}$ is convex as a function of $\Pi_{1}$, it can be easily confirmed that $f\left(\Pi_{1}+\Pi_{2}\right)$ is convex for a constant $\Pi_{2} \in \mathbb{R}^{N \times N}$.

c) if $f: \mathbb{R}^{N \times N} \rightarrow \mathbb{R}^{N \times N}$ is convex as a function of $\Pi_{1}$, it can be easily shown that $\Psi^{T} f\left(\Pi_{1}\right) \Psi$ is convex for an arbitrary matrix $\Psi \in \mathbb{R}^{N \times M}$.

Lemma 4: Let $\Pi_{1}$ and $\Pi_{2} \in \mathbb{R}^{N \times N}$ represent symmetric positive definite matrices. Let $f: \mathbb{R}^{N \times N} \rightarrow \mathbb{R}^{N \times N}$ represent the following function $f\left(\Pi_{1}\right)=\Pi_{1}\left(\Pi_{2}+\Pi_{1}\right)^{-1} \Pi_{1} . f$ is a convex function of $\Pi_{1}$.

proof:

$$
\begin{aligned}
f\left(\Pi_{1}\right) & =\Pi_{1}\left(\Pi_{2}+\Pi_{1}\right)^{-1} \Pi_{1} \\
& =\left[I_{N}-\Pi_{2}\left(\Pi_{2}+\Pi_{1}\right)^{-1}\right] \Pi_{1} \\
& =\Pi_{1}-\Pi_{2}+\Pi_{2}\left(\Pi_{2}+\Pi_{1}\right)^{-1} \Pi_{2} .
\end{aligned}
$$

Using Lemma 3, it can be easily seen that $f$ is a convex function of $\Pi_{1}$.

The following two lemmas relate stability region of scenario 3 to those of scenario 1 and 2 .

Lemma 5: The stability region of scenario 1 includes that of scenario 3: $\mu_{\text {ave,c,scenario } 1} \geq \mu_{\text {ave, }, \text {,scenario 3, where }}$ $\mu_{\text {ave }, c}$ represents the maximum tolerable average probability of packet loss for stability.

Proof: Consider a special case of scenario 1, where $R=0$. Let scenario 1 and scenario 3 have the same packet drop threshold. Let $P_{1}[k]$ and $P_{3}[k]$ represent the estimation error covariance matrices of scenario 1 with $R=0$ and scenario 3 respectively. Using Eq. 3 with $R=0$, we will have:

$$
\mathbb{E}\left(P_{1}[k+1]\right)=\mu_{\text {ave }} A \mathbb{E}\left(P_{1}[k]\right) A^{T}+Q .
$$

Consider $S_{1}[k]$ as it was defined in Section II-D. We will have

$\sigma_{z}^{2}[k] \succcurlyeq S_{1}[k] \Rightarrow \sigma_{z}^{2}[k]+C P_{3}[k] C^{T} \succcurlyeq S_{1}[k]+C P_{3}[k] C^{T}$.

Using Lemma $2 a$,

$$
\begin{aligned}
& A P_{3}[k] C^{T}\left(\sigma_{z}^{2}[k]+C P_{3}[k] C^{T}\right)^{-1} C P_{3}[k] A^{T} \preccurlyeq \\
& A P_{3}[k] C^{T}\left(S_{1}[k]+C P_{3}[k] C^{T}\right)^{-1} C P_{3}[k] A^{T} .
\end{aligned}
$$

Therefore,

$$
\begin{gathered}
P_{3}[k+1] \succcurlyeq A P_{3}[k] A^{T}- \\
A P_{3}[k] C^{T}\left(S_{1}[k]+C P_{3}[k] C^{T}\right)^{-1} C P_{3}[k] A^{T}+Q \Rightarrow \\
\mathbb{E}\left(P_{3}[k+1]\right) \succcurlyeq \mu_{\text {ave }} A \mathbb{E}\left(P_{3}[k]\right) A^{T}+Q,
\end{gathered}
$$

which results in the following:

$$
\text { if } \mathbb{E}\left(P_{3}[k]\right) \succcurlyeq \mathbb{E}\left(P_{1}[k]\right) \Rightarrow \mathbb{E}\left(P_{3}[k+1]\right) \succcurlyeq \mathbb{E}\left(P_{1}[k+1]\right) \text {. }
$$

Therefore the stability region of scenario 1 includes that of scenario 3 .
Lemma 6: The stability region of scenario 3 includes that of scenario $2: \mu_{\text {ave }, c \text {,scenario } 3} \geq \mu_{\text {ave }, c, \text { scenario } 2 \text {. }}$

Proof: Let $P_{2}[k]$ represent the estimation error covariance of scenario 2 for an $R \neq 0$, where no knowledge of $R$ is available in the estimator for scenario 2 (see footnote of Section III, part A). Using Eq. 6, $\mathbb{E}\left(P_{2}[k+1]\right)$ will be as follows:

$\mathbb{E}\left(P_{2}[k+1]\right)=\mu_{\text {ave }} A \mathbb{E}\left(P_{2}[k]\right) A^{T}+Q+A C^{-1} \Sigma C^{-1 T} A^{T}$,

where $\Sigma=\sigma_{n, \text { ave }}^{2} I_{N}+\left(1-\mu_{\text {ave }}\right) R$. Let $P_{3}[k]$ represent the estimation error covariance of scenario 3, as indicated by Eq. 24. We will have

$$
\begin{gathered}
\mathbb{E}\left(P_{3}[k+1] \mid P_{3}[k]\right)=\mu_{\text {ave }} \mathbb{E}\left(P_{3}[k+1] \mid P_{3}[k], \Upsilon[k] \leq \Upsilon_{T}\right) \\
+\left(1-\mu_{\text {ave }}\right) \mathbb{E}\left(P_{3}[k+1] \mid P_{3}[k], \Upsilon[k]>\Upsilon_{T}\right) .
\end{gathered}
$$

Using Lemma 3, it can be easily confirmed that $P_{3}[k+1]$ is a concave function of $\sigma_{z}^{2}[k]$ in Eq. 24. Therefore, using conditional Jensen's inequality, we will have,

$$
\begin{gathered}
\mathbb{E}\left(P_{3}[k+1] \mid P_{3}[k], \Upsilon[k]>\Upsilon_{T}\right) \preccurlyeq A P_{3}[k] A^{T}+Q- \\
A P_{3}[k] C^{T}\left(\mathbb{E}\left(\sigma_{z}^{2}[k] \mid \Upsilon[k]>\Upsilon_{T}\right)+C P_{3}[k] C^{T}\right)^{-1} C P_{3}[k] A^{T} .
\end{gathered}
$$

Therefore,

$\mathbb{E}\left(P_{3}[k+1] \mid P_{3}[k]\right) \preccurlyeq A P_{3}[k] A^{T}+Q-\left(1-\mu_{\text {ave }}\right) f\left(P_{3}[k]\right)$,

where $f: \mathbb{R}^{N \times N} \rightarrow \mathbb{R}^{N \times N}$ is as follows: $f\left(P_{3}[k]\right)=$ $A P_{3}[k]\left(C^{-1} \mathbb{E}\left(\sigma_{z}^{2}[k] \mid \Upsilon[k]>\Upsilon_{T}\right) C^{-1 T}+P_{3}[k]\right)^{-1} P_{3}[k] A^{T}$. It can be seen, using Lemma 4 , that $f$ is a convex function of $P_{3}[k]$. Therefore by applying Jensen's inequality,

$$
\begin{aligned}
& \mathbb{E}\left(P_{3}[k+1]\right) \preccurlyeq A \mathbb{E}\left(P_{3}[k]\right) A^{T}+Q-\left(1-\mu_{\text {ave }}\right) A \mathbb{E}\left(P_{3}[k]\right) C^{T} \\
& \quad \times\left[\mathbb{E}\left(\sigma_{z}^{2}[k] \mid \Upsilon[k]>\Upsilon_{T}\right)+C \mathbb{E}\left(P_{3}[k]\right) C^{T}\right]^{-1} C \mathbb{E}\left(P_{3}[k]\right) A^{T} .
\end{aligned}
$$

Noting that $\mathbb{E}\left(\sigma_{z}^{2}(\Upsilon[k]) \mid \Upsilon[k]>\Upsilon_{T}\right)=\frac{\Sigma}{1-\mu_{a v e}}$, it can be confirmed, after a few lines of derivations using Eq. 33 and 37 , that

if $\mathbb{E}\left(P_{3}[k]\right) \preccurlyeq \mathbb{E}\left(P_{2}[k]\right) \quad \Rightarrow \quad \mathbb{E}\left(P_{3}[k+1]\right) \preccurlyeq \mathbb{E}\left(P_{2}[k+1]\right)$.

Therefore the stability region of scenario 3 includes that of scenario 2.

Theorem 2: Consider the system model described in Section II. Consider a receiver that is equipped with a packet drop mechanism described by Eq. 1 but can support a crosslayer path. Then the estimation will be stable as long as the following holds: $\mu_{\text {ave, scenario } 3}<\rho_{\max }^{-2}$.

Proof: Lemma 5 and 6 showed that

$$
\mu_{\text {ave }, c, \text { scenario } 2} \leq \mu_{\text {ave }, c, \text { scenario } 3} \leq \mu_{\text {ave }, c \text {,scenario } 1 .} .
$$

Noting that scenario 1 and 2 have the same stability regions proves Theorem 2 .

Theorem 2 shows that availability of a cross-layer path does not impact the stability region. This suggests, similar to scenario 2 , that keeping all the packets will maximize the stability range.

2) Optimum Performance 
Theorem 3: Consider a receiver that is equipped with a packet drop mechanism described by Eq. 1 but can support a cross-layer path. Keeping all the packets, i.e. $\Upsilon_{T}=0$, will minimize the average estimation error covariance.

Proof: Let $P[k]$ represent the estimation error covariance of a receiver that is equipped with a cross-layer path, as indicated by Eq. 24. $P[k]$ can be written as follows using the same formulation utilized in the derivation of Eq. 27:

$P[k+1]=Q+A \Pi[k] A^{T}-A \Pi[k](P[k]+\Pi[k])^{-1} \Pi[k] A^{T}$,

where $\Pi[k]=C^{-1} \sigma_{z}^{2}[k] C^{-1 T}$. Let $P_{1}$ and $P_{2}$ represent estimation error covariance matrices of two estimators using $\Upsilon_{T 1}$ and $\Upsilon_{T 2}$, where $\Upsilon_{T 1}<\Upsilon_{T 2}$. Then for any received Signal to Noise Ratio at time step $k, \Upsilon[k]$, we will have

$$
\sigma_{z, 1}^{2}(\Upsilon[k]) \preccurlyeq \sigma_{z, 2}^{2}(\Upsilon[k]) \text { and } \Pi_{1}[k] \preccurlyeq \Pi_{2}[k],
$$

where $\sigma_{z, 1}^{2}$ and $\sigma_{z, 2}^{2}$ are as defined in Eq. 25 for these two estimators. Assume that $P_{1}[0]=P_{2}[0]$. It is easy to see that $P_{1}[1] \preccurlyeq P_{2}[1]$ for any $\Upsilon[0]$. Using Lemma 2 , the following can be easily confirmed for any given $\Upsilon[0], \Upsilon[1], \ldots, \Upsilon[k]$.

$$
\begin{gathered}
\text { if } P_{1}[k] \preccurlyeq P_{2}[k] \Rightarrow \\
-\left(P_{1}[k]+\Pi_{1}[k]\right)^{-1} \preccurlyeq-\left(P_{2}[k]+\Pi_{2}[k]\right)^{-1} \Rightarrow \\
\Pi_{1}[k]\left(P_{1}[k]+\Pi_{1}[k]\right)^{-1} \Pi_{1}[k] \succcurlyeq \\
\Pi_{2}[k]\left(P_{2}[k]+\Pi_{2}[k]\right)^{-1} \Pi_{2}[k] \\
\Rightarrow P_{1}[k+1] \preccurlyeq P_{2}[k+1] .
\end{gathered}
$$

This shows that using a lower threshold will result in a lower estimation error covariance. Therefore, keeping all the packets, i.e. $\Upsilon_{T}=0$, will minimize the estimation error covariance (and its average over the distribution of $\Upsilon$ ).

We can see that keeping all the packets not only prevents instability but also minimizes estimation error covariance in the presence of a cross-layer path.

To see the impact of a cross-layer path, Fig. 2 shows spectral norm of the average estimation error covariance after 300 time steps for the system parameters of Fig. 1 and for both scenario 2 and 3. By comparing the corresponding curves for these cases, it can be seen that a cross-layer path can improve the performance considerably even when compared to operating at the optimum $\Upsilon_{T}$ of scenario 2 . Furthermore, it can be seen that keeping more packets will reduce the norm of the estimation error covariance for scenario 3. In general, scenario 3 is more robust to the changes in $\Upsilon_{T}$ due to the availability of a cross-layer path, as can be seen from Fig. 2. Finally, the stability condition is confirmed to be the same as predicted by Theorem 2 .

\section{Summary And Further Extensions}

In this paper, we derived new design paradigms for estimating a multiple-input multiple-output dynamical system over mobile communication channels. We modeled the impact of the stochastic communication noise on the estimation process. We proved that in order to maximize the stability range, the receiver should keep all the packets independent of the quality of the link or availability of a cross-layer path. In the presence of a cross-layer path, this design will

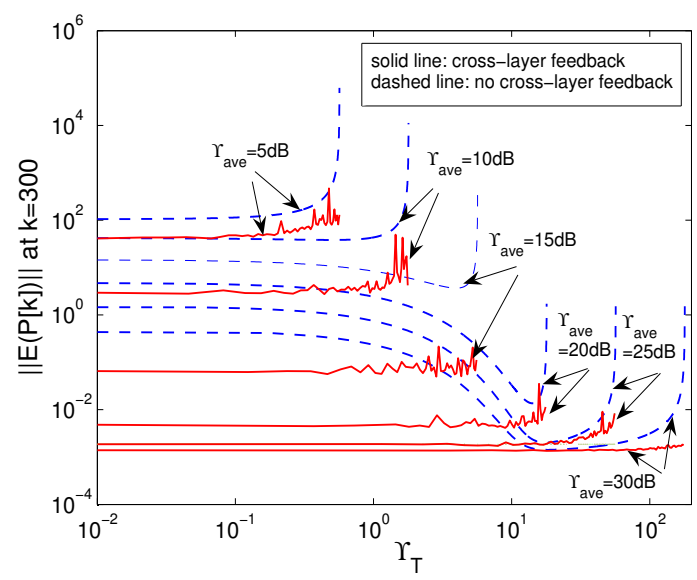

Fig. 2. Effect of a cross-layer path: compare scenario 2 and 3

also optimize the performance. However, in the absence of such a path, we proved that packet drop should be designed to balance information loss and communication noise in order to optimize the performance. The results can be easily extended to scenarios with multiple sensors by replacing Signal to Noise Ratio by Signal to Interference and Noise Ratio. Furthermore, the proposed design strategies should be applicable when considering the performance of a controller. Proving this analytically is among possible extensions of this work.

\section{REFERENCES}

[1] W. Jakes, Microwave Mobile Communications. IEEE Press, 1974

[2] D. Goodman, Wireless Personal Communications Systems. The Addison-Wesley Wireless Communications Series, 1997

[3] M. Micheli and M. I. Jordan, "Random Sampling of a Continuous-Time Dynamical Systems," 15th International Symposium on the Mathematical Theory of Networks and Systems (MTNS), 2002

[4] B.Sinopoli, L. Schenato, M. Franceschetti, K. Poolla, M. Jordan, S. Sastry, "Kalman filtering with intermittent observations," 42nd IEEE Conference on Decision and Control, 2003, vol: 1, pp: $701-708$

[5] Y. Mostofi and R. Murray, "On Dropping Noisy Packets in Kalman Filtering Over a Wireless Fading Channel," 24th American Control Conference (ACC), 2006

[6] Y. Mostofi and R. Murray, "New Design Principles for Estimation over Fading Channels in Mobile Sensor Networks," 44th IEEE Conference on Decision and Control (CDC), 2005

[7] D. Son, B. Krishnamachari and J. Heidemann, "Experimental Analysis of Concurrent Packet Transmissions in Low-Power Wireless Networks," USC-ISI Technical Report ISI-TR-2005-609, November 2005

[8] T. Kailath, A. H. Sayed and B. Hassibi, Linear Estimation. Prentice Hall information and system sciences series

[9] Y. Mostofi and R. Murray, "Effect of Time-Varying Fading Channels on the Control Performance of a Mobile Sensor Node," IEEE 1st International Conf. on Sensor and Adhoc Comm. and Networks, 2004

[10] S. Boyd and L. Vandenberghe, Convex Optimization. Cambridge University Press 2004

[11] R. Horn and C. Johnson, Matrix Analysis. Cambridge University Press 1999

[12] A. W. Marshall and I. Olkin, Inequalities: Theory of Majorization and Its Applications. Academic Press, New York, 1979 